\title{
Surgical ventricular reverse remodeling in severe ischemic dilated cardiomyopathy: The relevance of the left ventricular equator as a prognostic factor
}

Paolo Ferrazzi, MD, FETCS, ${ }^{\text {a }}$ Marco L. S. Matteucci, MD, ${ }^{a}$ Maurizio Merlo, MD, ${ }^{a}$ Attilio lacovoni, MD, ${ }^{a}$

Giuseppe Rescigno, MD, ${ }^{\text {a }}$ Matteo Bottai, PhD, ${ }^{\text {b }}$ Piervirgilio Parrella, $\mathrm{ScD},{ }^{\text {a }}$ Luca Lorini, MD, ${ }^{a}$ Michele Senni, MD, FESC, and Antonello Gavazzi, MD, FESC ${ }^{\mathrm{a}}$

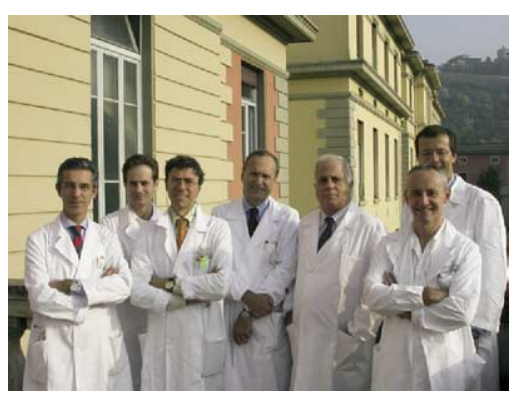

M. Senni, A. Iacovoni, M. Merlo, A. Gavazzi, P. Ferrazzi, L. Lorini (front), M. Matteucci (back) (left to right)

Supplemental material is available online.
From the Dipartimento Cardiovascolare Clinico e di Ricerca, ${ }^{a}$ Ospedali Riuniti Bergamo, Italy, and the Department of Epidemiology and Biostatistics, ${ }^{\mathrm{b}}$ Arnold School of Public Health, University of South Carolina, Columbia, SC.

Address for reprints: Paolo Ferrazzi, MD, Dipartimento Cardiovascolare Clinico e di Ricerca, Ospedali Riuniti, Largo Barozzi 1, 24100 Bergamo, Italy (E-mail: pferrazzi@ ospedaliriuniti.bergamo.it).

J Thorac Cardiovasc Surg 2006;131:357-63 $0022-5223 / \$ 32.00$

Copyright (C) 2006 by The American Association for Thoracic Surgery

doi:10.1016/j.jtcvs.2005.10.008
Objectives: Surgical ventricular reverse remodeling has been shown to possibly improve hemodynamics and symptoms, but effects on long-term mortality are not established. No consistent data are available on which patients will benefit most from this procedure. This study was designed to analyze the predictors of long-term survival after surgical ventricular reverse remodeling in patients with ischemic cardiomyopathy.

Methods: Eighty-five patients who underwent surgical ventricular reverse remodeling between May 1991 and October 2003 were retrospectively analyzed. Left ventricular wall motion and left ventricular equatorial diameter were assessed by means of angioventriculography. Left ventricular ejection fraction and volumes were measured by means of echocardiography. Cox regression analysis was used in several combinations to create a final model for identifying predictors of death.

Results: Actuarial survival after 1, 3, 5, and 10 years was $89 \%, 79 \%, 75 \%$, and $75 \%$, respectively. New York Heart Association class improved from $2.9 \pm 1.0$ to $1.3 \pm$ $0.5(P<.0001)$, left ventricular ejection fraction increased from $27.6 \% \pm 6.3 \%$ to $43.0 \% \pm 10.1 \%(P<.0001)$, and left ventricular end-systolic volume index decreased from $89.6 \pm 27.6 \mathrm{~mL} / \mathrm{m}^{2}$ to $56.5 \pm 34.5 \mathrm{~mL} / \mathrm{m}^{2}(P<.0001)$. Multivariate analysis identified left ventricular equatorial diameter of $70 \mathrm{~mm}$ or greater (hazard ratio, 5.28; 95\% confidence interval, $1.79-11.71 ; P=.020$ ) and segmental akinesia (hazard ratio, 4.46; 95\% confidence interval, $1.23-17.12 ; P=.024$ ) as the only independent predictors of death.

Conclusions: In this analysis of a single cohort of patients, surgical ventricular reverse remodeling improves the symptoms of ischemic cardiomyopathy, as well as left ventricular function, shape, and volume, with encouraging long-term outcomes, particularly in patients with dyskinesia. A left ventricular equatorial diameter of 70 $\mathrm{mm}$ or greater appears to be an important independent prognostic factor, which suggests the relevance of the left ventricular equatorial region for effective surgical reverse remodeling.

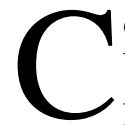

oronary artery disease is the most frequent cause of heart failure (HF) in Western countries. ${ }^{1}$ Despite improvements with medical treatment, the prognosis of patients with end-stage HF remains poor. ${ }^{2}$ Although heart transplantation is the best option for end-stage cardiomyopathy, this treatment is limited by a shortage of donors. Furthermore, in the subgroup with ischemic cardiomyopathy, both mortality on the waiting list and long-term results of heart transplantation are worse than in patients with idiopathic dilated cardiomyopathy. ${ }^{3,4}$ These factors contributed to the development of surgical techniques with the aim of improving the function of the left ventricle. Left ventricular (LV) surgical reverse 


\section{Abbreviations and Acronyms \\ $\mathrm{ACE}=$ angiotensin-converting enzyme \\ $\mathrm{HF} \quad=$ heart failure \\ $\mathrm{LV} \quad=$ left ventricular \\ LVEF = left ventricular ejection fraction \\ LVEqD = left ventricular end-diastolic equatorial diameter \\ LVESVI $=$ left ventricular end-systolic volume index \\ MLHF = Minnesota Living With Heart Failure \\ NYHA $=$ New York Heart Association \\ SVR = surgical ventricular reverse remodeling}

restoration (surgical ventricular reverse remodeling [SVR]; surgical remodeling and ventricular reshaping) or surgical ventricular restoration ${ }^{5}$ has been found to improve symptoms and ventricular function in patients with ischemic cardiomyopathy. ${ }^{6,7}$ This operative method benefits patients with akinesia or dyskinesia of anterior LV segments and decreased LV function by reducing ventricular wall stress and restoring the spherical left ventricle to a more physiologic elliptical shape. ${ }^{8}$ A recent registry of 1198 SVR procedures performed in 13 centers worldwide indicates encouraging results. ${ }^{9}$ In this study different surgical teams used a variety of surgical techniques, and follow-up time was limited to 5 years. The ongoing Surgical Treatment for Ischemic Heart Failure trial was designed to address open and controversial issues about SVR in patients with HF. ${ }^{10}$ We thought it would be worth reviewing our series of SVR before we entered this trial in November 2003 to identify any factors that could help in predicting outcomes. Because this series represents our whole 12-year experience with SVR, it might also provide some useful long-term follow-up information.

\section{Methods}

\section{Patient Population}

Between May 1991 and October 2003, 122 patients with LV dysfunction caused by coronary artery disease underwent SVR. Excluded from the analysis were patients with posterior akinetic or dyskinetic LV segments $(\mathrm{n}=18)$, patients with an LV ejection fraction (LVEF) of greater than $35 \%(n=10)$, patients undergoing the Batista procedure $(n=4)$, patients with an associated aortic valve replacement $(\mathrm{n}=2)$, patients with postinfarction ventricular septal defect closure $(\mathrm{n}=2)$, and a child previously operated on for arterial switch repair for transposition of the great arteries with a postischemic LV aneurysmectomy. The remaining 85 patients were the subjects of the study.

Functional status was evaluated by using the New York Heart Association (NYHA) classification, and quality of life was assessed through a self-administered Minnesota Living With Heart Failure (MLHF) questionnaire. From August through November 2003, survivors were invited to take part in a clinical survey, which included an echocardiographic evaluation. All deaths were classi- fied on the basis of the available information (hospital records, death certificates, and statements from witnesses). Death was considered caused by progressive HF in cases of onset and progression of symptoms of definite HF. Sudden death was defined as death within 1 hour of new symptoms or witnessed death without new symptoms in the 72 hours preceding death. In-hospital mortality included any death occurring during hospitalization.

\section{Echocardiography}

Echocardiographic studies were performed with a $2.5-$ or $3.5-\mathrm{MHz}$ transducer. Mitral regurgitation was assessed semiquantitatively as grade $1+$ to $4+$ by means of color flow Doppler scanning. LVEF and LV volumes were calculated by using a modified version of Simpson's rule. ${ }^{11}$ Preoperative data were obtained by reviewing reports. Follow-up assessment consisted of a complete 2-dimensional and Doppler echocardiographic study performed by a single operator who had no knowledge of the patients' clinical status.

\section{Cardiac Catheterization}

All patients underwent preoperative ventricular angiography in the standard $30^{\circ}$ right anterior oblique projection with injection of 0.7 $\mathrm{mL} / \mathrm{kg}^{-1}$ nonionic contrast medium at a rate of $12 \mathrm{~mL}^{\mathrm{s}-1}$ filmed at $50 \mathrm{frames}^{\mathrm{s}-1}$. Ventricular volumes were calculated by using the Chapman monoplane method, ${ }^{12}$ and anterior regional wall motion was analyzed with the centerline method ${ }^{13}$ to distinguish akinetic and dyskinetic segments. Regional wall motion was defined as dyskinetic if the absolute motion of contiguous chords was less than zero and akinetic if it was equal to zero. The LV end-diastolic equatorial diameter ( $\mathrm{LVEqD}$ ) was measured as the $\mathrm{LV}$ transversal diameter at the midpoint of the longitudinal axis between the mitral plane and the LV apex on end-diastolic angioventriculographic frames. The mean of 3 measurements obtained during 3 different cardiac cycles was calculated for each patient and used for the subsequent statistical analysis.

\section{Operative Technique}

The aim of the operation was to exclude all asynergic areas of the ventricle, restoring a more physiologic elliptical geometry and LV volume. All patients underwent SVR either through the Dor procedure or a modified Dor-type technique (without the Dacron patch). ${ }^{14-16}$

Coronary artery bypass grafting, mitral valve repair or replacement, biventricular pacing, and/or automatic cardioverter device implantation were carried out as necessary. A single surgeon performed most $(82 \%)$ of the operations.

\section{Statistical Analysis}

Results are presented as mean values \pm standard deviation or frequency percentages. Baseline and follow-up findings were compared by using the Wilcoxon signed-rank nonparametric test for continuous variables and the Fisher exact test for categoric variables. Because of the relatively small size of the sample and few events, we estimated several Cox proportional hazard models of analysis (no more than 6 variables at a time), removing and adding each variable as a stepwise method to maximize the explained variance of the model. At the end, we found 4 variables with a cutoff point of a $P$ value of .020 for further analyses (ejection fraction, LVEqD, MLHF score, and akinesia-dyskinesia). A final 


\section{TABLE 1. Patient characteristics}

\begin{tabular}{lc}
\hline Age, y (range) & $60.5 \pm 9(37-78)$ \\
Male/female sex & $70 / 15$ \\
Prevalent HF, no. of patients (\%) & $71(83.5)$ \\
Angina, no. of patients (\%) & $46(54.1)$ \\
Isolated angina & $14(16.5)$ \\
Angina + prevalent HF & $32(37.6)$ \\
Mean NYHA class & $2.9 \pm 1.0$ \\
NYHA class IV, no. of patients (\%) & $26(30.6)$ \\
LVEF \% (range) & $27.6 \pm 6.3(14-35)$ \\
LVESVI >90 mL/m², no. of patients (\%) & $47(55.3)$ \\
Mitral regurgitation (moderate-severe), & $16(18.9)$ \\
$\quad$ no. of patients (\%) & \\
Dyskinesia/akinesia & $46 / 39$
\end{tabular}

$H F$, Heart failure; $N Y H A$, New York Heart Association; $L V E F$, left ventricular ejection fraction; LVESVI, left ventricular end-systolic volume index.

model was used to assess the independent prognostic value of the selected significant variables at long-term mortality. Hazard ratios and $95 \%$ confidence intervals were calculated. The Kaplan-Meier survival curves were constructed for the significant predictors of outcome, and differences were tested by using the log-rank test. Statistical analysis was performed with SAS version 8.2 (SAS Institute, Inc, Cary, NC) and SPSS version 9.0 (SPSS Inc, Chicago, Ill) software.

\section{Results}

\section{Study Population}

The characteristics of the 85 patients are summarized in Table 1 and Appendix E1. Fifty-six (65.9\%) patients were in NYHA functional class III or IV. Ventricular volumes were severely enlarged. Most of the patients underwent associated procedures: coronary artery bypass grafting in 82 (96.5\%) patients and mitral valve correction in $16(18.8 \%)$ patients (repair in 15 and replacement in 1). Biventricular pacing, automatic cardioverter device implantation, or both were carried out in $2(2.4 \%)$ patients.

\section{Clinical Outcomes}

Six $(7.1 \%)$ patients died in the hospital: 1 during the operation and 5 in the intensive care unit a mean of 6.5 days after the operation. The causes of death were low output syndrome in 4 patients and associated ventricular arrhythmia in 2 patients. The mean intensive care unit length of stay was $4.4 \pm 7.6$ days.

The 79 patients discharged were followed up for a mean of 43.7 months (range, 3-144 months). During this period, 14 patients died after a mean of 741 days: the causes of death were progressive HF $(n=5)$, sudden death $(n=4)$, acute myocardial infarction $(\mathrm{n}=1)$, ruptured abdominal aortic aneurysm $(\mathrm{n}=1)$, pneumonia $(\mathrm{n}=1)$, cancer $(\mathrm{n}=$ $1)$, and septic shock $(\mathrm{n}=1)$. None of the patients underwent transplantation. The survival rates after $1,3,5$, and 10 years were $89 \%, 79 \%, 75 \%$, and $75 \%$, respectively. There were no sex differences in outcomes.
TABLE 2. Hazard ratio of prognostic factors affecting survival

\begin{tabular}{lccc}
\hline & Hazard ratio & $\boldsymbol{P}$ Values & $\begin{array}{c}\text { 95\% Confidence } \\
\text { interval }\end{array}$ \\
\hline LVEqD & 5.28 & $>.020$ & $1.79-11.71$ \\
MLHF & 0.93 & $>.1742$ & $0.070-1.06$ \\
LVEF & 1.01 & $>.2748$ & $0.86-1.13$ \\
Akinesia & 4.46 & $>.024$ & $1.23-17.12$
\end{tabular}

$\angle V E q D$, Left ventricular equatorial diastolic diameter; $M L H F$, minnesota living with heart failure score; $L V E F$, left ventricular ejection fraction.

A symptomatic improvement was noted in most of the survivors: the NYHA functional class decreased from $2.9 \pm$ 1.0 to $1.3 \pm 0.5(P<.0001)$, and the MLHF score decreased from $44.2 \pm 21.5$ to $24.1 \pm 15.5(P<.0001)$.

\section{Preoperative Predictors of Survival}

The enlarged LVEqD had an inverse proportional relationship with the outcome of SVR. Although the equator is a continuous variable, the logistic analysis showed, among the other possible figures, a bifurcation into less than $70 \mathrm{~mm}$ and greater than $70 \mathrm{~mm}$, which was particularly significant as a determinant of prognosis $(P<.0001)$. Amid all of the diameters and volumes, an LVEqD of $70 \mathrm{~mm}$ or greater was the most important predictor of mortality, increasing the hazard of death by approximately 4.5 times. Segmental akinesia was also a major predictor of outcome, with a 3.5-time increase in the risk of death (Table 2).

Survival at each time point was significantly better in patients with an LVEqD of less than $70 \mathrm{~mm}$ and in patients with dyskinesia versus akinesia (Figure 1). The association of an enlarged LVEqD and segmental akinesia carried the poorest prognosis: survival in this group was $18 \%$ versus $88 \%$ in the group with dyskinesia and an LVEqD of less than $70 \mathrm{~mm}$. Mitral valve surgery was not a risk factor $(P=.267)$.

\section{Postoperative LV Reverse Remodeling}

The 65 survivors were contacted from November 2003 through February 2004. Mean echocardiographic LVEF improved from $27.6 \% \pm 6.3 \%$ to $43.0 \% \pm 10.1 \%(P<$ $.0001)$. LV end-diastolic volume index decreased from $131.6 \pm 34.7$ to $92.8 \pm 51.2 \mathrm{~mL} / \mathrm{m}^{2}(P<.0001)$, and $\mathrm{LV}$ end-diastolic diameter index decreased from $35.3 \pm 5.5$ to $33.0 \pm 13.7 \mathrm{~mm} / \mathrm{m}^{2}(P<.0001)$. Furthermore, LV endsystolic volume index (LVESVI) decreased from $89.6 \pm$ 27.6 to $56.5 \pm 34.5 \mathrm{~mL} / \mathrm{m}^{2}(P<.0001)$, and $\mathrm{LV}$ endsystolic diameter index decreased from $28.14 \pm 5.15$ to $26.9 \pm 12.6 \mathrm{~mm} / \mathrm{m}^{2}(P=.04)$. These favorable changes were less marked and did not reach statistical significance among the patients in the highest LVEqD quartile $(\geq 70$ $\mathrm{mm}$ ), whereas they were highly significant in those with an 


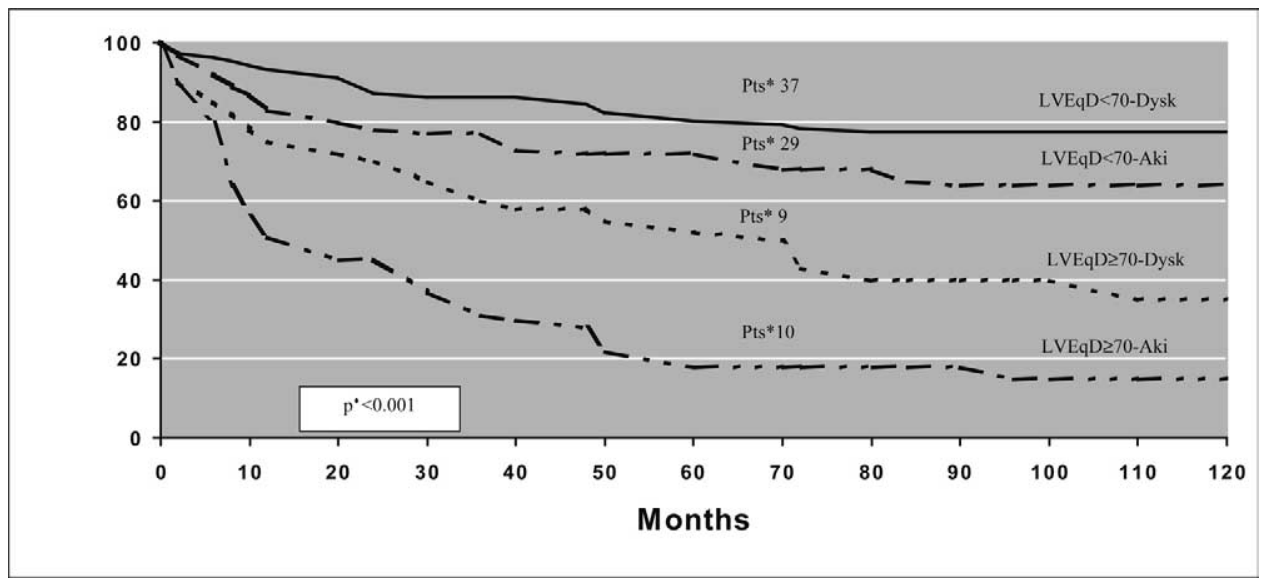

Figure 1. Survival curves: dyskinetic with low versus high left ventricular end-diastolic equatorial diameter and akinetic with low versus high left ventricular end-diastolic equatorial diameter. Pts, Patients at risk; $P$, log-rank test for all groups.

LVEqD of less than $70 \mathrm{~mm}$ (Table 3). Conversely, LV volumes decreased similarly in the presence of akinesia or dyskinesia (Table 4).

\section{Discussion}

SVR in ischemic cardiomyopathy is a procedure refined over the years, ${ }^{6,717}$ the retrospective evaluations of which demonstrated a significant clinical benefit over time. ${ }^{9,18}$ Our data confirm the effectiveness of SVR. The majority of patients reported improvement in quality of life with a $75 \%$ survival after 10 years; however, the efficacy of SVR in ischemic cardiomyopathy versus medical therapy is debated. In the last 2 decades, randomized clinical trials have documented the beneficial effects on survival of angiotensin-converting enzyme (ACE) inhibitors, $\beta$-blockers, aldosterone antagonists, and angiotensin receptor blockers in patients with HF and decreased LVEF. Recent pathophysiologic evidence supported the concepts that LV remodeling is related to clinical outcome and that ACE inhibitors, ${ }^{19}$ AT1 receptor antagonists, ${ }^{20}$ and $\beta$-blockers ${ }^{21-24}$ slow or reverse the progression of ventricular remodeling. However, available data on reverse remodeling in ischemic cardiomyopathy are limited to 18 months of treatment and document reductions in LV end-systolic volume in a mean range of $12.7 \% .^{21-24}$

The reverse remodeling with the surgical approach in conjunction with medical therapy led in the Reconstructive Endoventricular Surgery returning Torsion Original Radius Elliptical shape to the left ventricle (RESTORE) study to a decrease in LVESVI of $36 \%$ of the preoperative values. Eighty-six percent of our patients were taking ACE inhibitors and $66 \%$ were taking $\beta$-blockers at follow-up, and the decrease in LVESVI was 37\%. Although the influence of medical treatment on survival and remodeling remains an unresolved factor, our data highlight the relevance for surgical outcome of the equatorial zone of the left ventricle and stress the negative synergism between an increased LVEqD and segmental akinesia.

The relevance of the equatorial zone of the left ventricle finds theoretic support in studies concerning the structural changes that take place in ischemic cardiomyopathy during acquisition of spherical shape in the failing heart. We are

TABLE 3. Comparison of preoperative and follow-up clinical and echocardiographic characteristics by left ventricular equatorial diameter

\begin{tabular}{|c|c|c|c|c|c|c|}
\hline & \multicolumn{3}{|c|}{ LVEqD $<70 \mathrm{~mm}$} & \multicolumn{3}{|c|}{ LVEqD $\geq 70 \mathrm{~mm}$} \\
\hline & Preoperative & Follow-up & $P$ value* & Preoperative & Follow-up & $P$ value * \\
\hline NYHA & $2.8 \pm 1.0$ & $1.3 \pm 0.5$ & $<.0001$ & $2.9 \pm 1.1$ & $1.5 \pm 0.8$ & .026 \\
\hline MLHF score & $49.3 \pm 21.7$ & $23.9 \pm 16.8$ & $<.0001$ & $42.7 \pm 25.2$ & $25.7 \pm 18.6$ & .16 \\
\hline LVEF, \% & $27.5 \pm 6.1$ & $43.4 \pm 10.6$ & $<.0001$ & $22.4 \pm 6.4$ & $38.4 \pm 11.2$ & .002 \\
\hline LVEDDI, $\mathrm{mm} / \mathrm{m}^{2}$ & $34.4 \pm 5.9$ & $31.2 \pm 4.3$ & .002 & $38.7 \pm 5.4$ & $42.1 \pm 32.4$ & .17 \\
\hline LVESDI, $\mathrm{mm} / \mathrm{m}^{2}$ & $27.1 \pm 5.6$ & $25.0 \pm 5.8$ & .01 & $31.7 \pm 5.1$ & $35.7 \pm 27.1$ & .79 \\
\hline LVEDVI, mL/m² & $124.2 \pm 30.3$ & $87.4 \pm 29.2$ & $<.0001$ & $157.2 \pm 39.8$ & $117.5 \pm 105.9$ & .10 \\
\hline LVESVI, $\mathrm{mL} / \mathrm{m}^{2}$ & $83.3 \pm 22.7$ & $52.4 \pm 23.7$ & $<.0001$ & $111.4 \pm 34.1$ & $77.2 \pm 66.1$ & .09 \\
\hline
\end{tabular}

$\angle V E q D$, Left ventricular equatorial diastolic diameter; NYHA, New York Heart Association; MLHF, Minnesota living with heart failure; $L V E F$, left ventricular ejection fraction; LVEDDI, left ventricular end-diastolic diameter index; LVESDI, left ventricular end-systolic diameter index; LVEDVI, left ventricular end-diastolic volume index; LVESVI, left ventricular end-systolic volume index. *Wilcoxon signed-rank test. 
TABLE 4. Comparison of preoperative and follow-up clinical and echocardiographic characteristics by segmental wall motion status

\begin{tabular}{|c|c|c|c|c|c|c|}
\hline & \multicolumn{3}{|c|}{ Dyskinesia } & \multicolumn{3}{|c|}{ Akinesia } \\
\hline & Preoperative & Follow-up & $P$ value* & Preoperative & Follow-up & $P$ value* \\
\hline NYHA & $2.6 \pm 1.0$ & $1.2 \pm 0.5$ & $<.0001$ & $2.9 \pm 1.0$ & $1.5 \pm 0.5$ & $<.0001$ \\
\hline MLHF score & $45.8 \pm 21.3$ & $23.0 \pm 16.7$ & $<.0001$ & $46.8 \pm 19.9$ & $26.1 \pm 16.4$ & $<.0001$ \\
\hline LVEF, \% & $28.5 \pm 6.1$ & $44.5 \pm 11.6$ & $<.0001$ & $25.9 \pm 6.4$ & $39.5 \pm 9.5$ & $<.0001$ \\
\hline LVEDDI, mm/m² & $34.7 \pm 6.4$ & $33.3 \pm 17.4$ & .61 & $35.5 \pm 4.6$ & $32.5 \pm 4.9$ & .01 \\
\hline LVESDI, mm/m² & $27.4 \pm 6.2$ & $26.6 \pm 15.5$ & .74 & $28.6 \pm 4.7$ & $27.3 \pm 4.1$ & .74 \\
\hline LVEDVI, mL/m² & $130.0 \pm 40.9$ & $91.2 \pm 37.7$ & $<.0001$ & $129.1 \pm 30.9$ & $88.8 \pm 40.9$ & $<.0001$ \\
\hline LVESVI, mL/m² & $86.4 \pm 32.1$ & $55.5 \pm 40.5$ & $<.0001$ & $89.6 \pm 21.5$ & $58.2 \pm 23.3$ & $<.0001$ \\
\hline
\end{tabular}

$\angle V E q D$, Left ventricular equatorial diastolic diameter; $N Y H A$, New York Heart Association; $M L H F$, Minnesota living with heart failure; $L V E F$, left ventricular ejection fraction; LVEDDI, left ventricular end-diastolic diameter index; LVESDI, left ventricular end-systolic diameter index; LVEDVI, left ventricular end-diastolic volume index; LVESVI, left ventricular end-systolic volume index. *Wilcoxon signed-rank test.

addressing the vicious cycle relating to the changes in myofibril orientation from oblique to transverse disposition caused by the loss of elliptical shape that results in a diminishing function, which in turn causes more enlargement with deeper geometric (spherical) consequence. ${ }^{25}$ This plays a complementary negative role to the augmented cardiac wall stress caused by the enlarged chamber, with all the well-known adverse consequences. Thus far, the sphericity index could have a prominent part in candidates for SVR, and LVEqD can be a surrogate that reflects changes toward sphericalization of the left ventricle. ${ }^{26}$ In line with this, we found that preoperative $\mathrm{LVEqD}$ correlated best with outcome, whereas LV internal end-diastolic diameter (measured by means of echocardiography at the level of the basal segments) was not significantly related to prognosis. Among all of the LV diameters and volumes, LVEqD was the only independent factor of clinical outcome. In patients with an LVEqD of $70 \mathrm{~mm}$ or greater, the inconsistent changes in volumes and diameters underline the unpredictability of LV remodeling in such cases. We wonder whether a certain value of LVEqD could represent a point of no return for SVR. The Dor procedure, although reducing the longitudinal axis while reshaping the apex, does not significantly reduce the short axis (equatorial diameter), despite the oblique position of patch toward the septum,,${ }^{6,7,27,28}$ and hence does not positively affect the sphericity index of the left ventricle. The enlarged LVEqD could possibly be the Achilles' heel of the Dor procedure.

Other inferences, with clinical consequences, can be drawn from geometric considerations related to LVEqD more than to the LV volume itself. The ventricular equator is the region in which maximum tension is generated during systole and is also the fulcrum of the twisting and untwisting of myocardial fibers. ${ }^{29-31}$ Moreover, because the papillary muscles are inserted in the equatorial region, they tend to migrate laterally in a spherical ventricle and cause ensuing functional mitral regurgitation. ${ }^{26}$ On the other end, a number of surgical series have indicated preoperative LV volumes as one of the most important determinants of clinical outcome. ${ }^{32,33}$ The RESTORE investigators have recently stressed the prognostic relevance of preoperative LV volumes. ${ }^{9}$ In this study the conventional predictors of HF outcome (advanced age, functional class, and LVEF, together with LVESVI) were predictive of survival. Conversely, in our analysis taking into account $\mathrm{LVEqD}$, as we saw in the correlation matrix, neither LV volume nor LVEF was an independent risk factor. A reasonable explanation for this discrepancy is that at similarly increased volumes, uniform or regional remodeling might be the substrate of different ventricular shapes and lead to different outcomes. Furthermore, we could speculate that reductions in LVEF observed in our patients could be related more to the loss of proper myocardial fiber geometric orientation than to real contraction impairment.

Concerning the second issue, the negative synergism between the increased LVEqD and segmental akinesia, previous studies have reported better results or no difference in outcome between patients with dyskinetic and those with akinetic segments. ${ }^{6,7,9,34-36}$ Successful SVR comes from the effective exclusion of the nonfunctional area, a process that is critically dependent on demarcation. Although it has been suggested that the extent of the scar is more important than the type of wall motion (asynergia or dyskinesia), ${ }^{6}$ in our patients an akinetic segment was an independent predictor of poor outcome, with a 4-fold increase in the risk of death. Dyskinetic scar and akinetic tissue both indicate abnormal conditions characterized by the absence of functional tissue, but surely there is less of a consensus regarding the physiologic consequences of volume reduction through exclusion of akinetic wall segments. After myocardial infarction, the material properties of the affected wall vary. This is particularly true after the advent of thrombolysis, direct percutaneous transluminal angioplasty, or both that more frequently cause subendocardial necrosis or patchy trabecular 
scarring, leading to segmental akinesia. The interaction between this akinetic region with the rest of the myocardium could be less predictable than that of the compliant dyskinetic scar, which should explain the reported equivocal effect of akinetic scar resection on systolic and diastolic properties. ${ }^{36}$ Although an independent predictor of outcome, akinesia was most deleterious in patients with an enlarged LVEqD, delimiting a subgroup with a particularly poor prognosis.

In conclusion, this study included a broad spectrum of patients ranging from classical $\mathrm{LV}$ aneurysm resection (dyskinetic) and resection of nonaneurysmal (akynetic) LV wall muscle in severely dilated chamber. Even if, in this setting, the term SVR (especially in the past) could be misleading, it includes a grey area where is difficult to foresee the degree of dilation residual to surgical resection. Hence LVEqD might preoperatively help in estimating the residual left ventricle and could also contain part of the answer to the tantalizing dilemma of having the same preoperative volumes but different outcome.

\section{Study Limitations}

Ours was a retrospective cohort analysis and has the inherent limitations of this design. The study population was relatively small, but the group was homogeneous, and the majority of patients presented with advanced HF.

The angiographic assessment of LVEqD is invasive and was not performed during the follow-up. Preoperative echocardiographic analyses of LVEqD were not available because the echocardiographic assessments were not recorded in most cases. We acknowledge that the postoperative LVEqD measurement would be extremely important for drawing conclusions regarding the surgical objective of treating the form and not just the evident disease. At the same time, we understand that this study might be of some value as a stimulus for further investigations.

We did not have any consistent information concerning preoperative myocardial viability. Although the majority of the patients had undergone radionuclide scanning or an echocardiographic stress test, the techniques used over the years were so disparate that it was decided to exclude this evaluation from the analysis.

\section{Conclusions}

The results of this study provide further evidence of the effectiveness of SVR in patients with severe ischemic cardiomyopathy. A careful preoperative assessment might identify the optimal candidates for this procedure, and the ongoing Surgical Treatment for Ischemic Heart Failure trial will provide more insights into this intriguing matter. We retrospectively assessed the ability of LVEqD, a reliable and easy-to-assess parameter, to affect survival, particularly when associated with segmental akinesia. For our patients with severe ischemic dilated cardiomyopathy, an LVEqD of less than $70 \mathrm{~mm}$ anticipated successful SVR. Surgical maneuvers in ischemic cardiomyopathy have thus far corrected LV apex abnormalities by means of SVR and LV base dilatation by using undersized mitral rings. Our results suggest the importance of adding the LVeqD measurements to our diagnostic criteria and considering the LV equatorial region a possible target of surgical correction.

\section{References}

1. Gheorghiade M, Bonow RO. Chronic heart failure in the United States: a manifestation of coronary artery disease. Circulation. 1998;97:282-9.

2. Senni M, Tribouilloy CM, Rodeheffer RJ, et al. Congestive heart failure in the community: trends in incidence and survival in a 10-year period. Arch Intern Med. 1999;159:29-34.

3. Stevenson LW, Tillisch JH, Hamilton M, et al. Importance of hemodynamic response to therapy in predicting survival with ejection fraction $20 \%$ secondary to ischemic or nonischemic dilated cardiomyopathy. Am J Cardiol. 1990;66:1348-52.

4. Cimato TR, Jessup M. Recipient selection in cardiac transplantation: contraindications and risk factors for mortality. J Heart Lung Transplant. 2002;21:1161-73.

5. Dor V. Left ventricular reconstruction: the aim and the reality after twenty years. J Thorac Cardiovasc Surg. 2004;128:17-20.

6. Di Donato M, Sabatier M, Dor V, Toso A, Maioli M, Fantini F. Akinetic versus dyskinetic postinfarction scar: relation to surgical outcome in patients undergoing endoventricular circular patch plasty repair. J Am Coll Cardiol. 1997;29:1569-75.

7. Mickleborough LL, Merchant N, Ivanov J, Rao V, Carson S. Left ventricular reconstruction: early and late results. J Thorac Cardiovasc Surg. 2004;128:27-37.

8. Di Donato M, Sabatier M, Dor V, et al. Effects of the Dor procedure on left ventricular dimension and shape and geometric correlates of mitral regurgitation one year after surgery. J Thorac Cardiovasc Surg. 2001;121:91-6.

9. Athanasuleas CL, Buckberg GD, Stanley AW, et al . Surgical ventricular restoration in the treatment of congestive heart failure due to post-infarction ventricular dilation. J Am Coll Cardiol. 2004;44: $1439-45$.

10. Jones RH. The year in cardiovascular surgery. J Am Coll Cardiol. 2004;43:1706-14.

11. Schiller NB, Shah PM, Crawfor M, et al. Recommendation for quantification of the left ventricle by 2-dimensional echocardiography. J Am Soc Echocardiogr. 1989;2:358-67.

12. Chapman CB, Baker O, Reynolds J, Bonte FJ. Use of biplane cinefluorography for measurement of ventricular volume. Circulation. 1958; 18:1105-17.

13. Sheehan FH, Bolson EL, Dodge HT, Mathey DG, Schofer J, Woo HW. Advantages and applications of the centerline method for characterizing regional ventricular function. Circulation. 1986;74:293-305.

14. Jatene AD, Paulista PP, Souza LC, et al. [Jatene's operation. Technique, indications and current results]. Arq Bras Cardiol. 1983;41:303-8.

15. Guilmet D, Popoff G, Dubois C, et al. [A new surgical technique for the treatment of left ventricular aneurysm: overcoat aneurysmoplasty. Preliminary results. 11 cases]. Arch Mal Coeur Vaiss. 1984;77:953-8.

16. Dor V, Saab M, Coste P, Kornaszewska M, Montiglio F. Left ventricular aneurysm: a new surgical approach. Thorac Cardiovasc Surg. 1989;37:11-9.

17. Caldeira C, McCarthy PM. A simple method of left ventricular reconstruction without patch for ischemic cardiomyopathy. Ann Thorac Surg. 2001;72:2148-9.

18. Westaby S. Surgical restoration of the failing left ventricle. Med Clin North Am. 2003;87:523-52.

19. Greenberg B, Quinones MA, Koilpillai C et al. Effects of long-term enalapril therapy on cardiac structure and function in patients with left ventricular dysfunction. Results of the SOLVD echocardiographic substudy. Circulation. 1995;91:2573-81. 
20. Wong M, Staszewsky L, Latini R, Barlena S, et al. Valsartan benefits left ventricular structure and function in heart failure: Val-HeFT echocardiographic study. J Am Coll Cardiol. 2002;40:970-5.

21. Groenning BA, Nilsson JC, Sondergaard L, et al. Antiremodelling effects on the left ventricular during beta blockade with metoprolol in the treatment of chronic heart failure. J Am Coll Cardiol. 2000;36:2072-80.

22. Doughty RN, Whalley G, Gamble G, et al. Left ventricular remodelling with carvedilol in patients with congestive heart failure due to ischemic heart disease. J Am Coll Cardiol. 1997;29:1060-6.

23. Hall SA, Cigarroa CG, Marcoux L, et al. Time course of improvement in left ventricular function, mass and geometry in patients with congestive heart failure treated with beta-adrenergic blockade. J Am Coll Cardiol. 1995;25:1154-61.

24. Doughty RN, Whalley Ga, Walsh HA, et al. Effects of carvedilol on left ventricular remodelling after acute myocardial infarction: the CAPRICORN Echo substudy. Circulation. 2004;109:201-6.

25. Buckberg GD. Basic science review: the helix and the heart. J Thorac Cardiovasc Surg. 2002;124:863-83.

26. Kono T, Sabbah HN, Rosman H, Alam M, Jafri S, Goldstein S. Left ventricular shape is the primary determinant of functional mitral regurgitation in heart failure. J Am Coll Cardiol. 1992;20:1594-8.

27. Menicanti L, Di Donato M. The Dor procedure: what has changed after fifteen years of clinical practice? J Thorac Cardiovasc Surg. 2002; 124:886-90.
28. Dor V. Left ventricular aneurysms: the endoventricular circular patch plasty. Semin Thorac Cardiovasc Surg. 1997;9:123-30.

29. Ingels NB. Myocardial fiber architecture and left ventricular function. Technol Health Care. 1997;5:45-52.

30. Ingels NB, Daughters GT, Stinson EB, Aldermann E. Measurement of midwall myocardial dynamics in intact man by radiography of surgically implanted markers. Circulation. 1975;52:859-67.

31. Spotnitz HM. Macro design, structure, and mechanics of the left ventricle. J Thorac Cardiovasc Surg. 2000;119:1053-77.

32. Yamaguchi A, Ino T, Adachi H, et al. Left ventricular volume predicts postoperative course in patients with ischemic cardiomyopathy. Ann Thorac Surg. 1998;65:434-8.

33. Buckberg GD. Congestive heart failure: treat the disease, not the symptom-return to normalcy. J Thorac Cardiovasc Surg. 2001;121: 628-37.

34. Couper GS, Bunton RW, Birijiniuk V, et al. Relative risks of left ventricular aneurysmectomy in patients with akinetic scars versus true dyskinetic aneurysms. Circulation. 1990;82(suppl IV):248-56.

35. Mangshau A. Akinetic versus dyskinetic left ventricular aneurysms diagnosed by gated scintigraphy: differences in surgical outcome. Ann Thorac Surg. 1989;47:746-51.

36. Atrip JH, Oz MC, Burkoff D. Left ventricular volume reduction surgery for heart failure: a physiologic perspective. J Thorac Cardiovasc Surg. 2001;122:775-82.

\section{JTCVS On-Line Manuscript Submission and Review}

The Journal of Thoracic and Cardiovascular Surgery requires authors and reviewers to submit all new and revised manuscripts and reviews via Editorial Manager. Point your browser to http://jtcvs.editorialmanager.com, log in as author or reviewer (as appropriate), and follow the instructions provided.

To retrieve your username and password, click "Forget your password?" on the Editorial Manager log-in page.

If you have questions or experience problems uploading your manuscript or review, please contact the editorial office:

Telephone: 215-762-1854

E-mail: jtcvs@drexel.edu 


\section{APPENDIX E1}

Patient characteristics and stepwise regression for variable selection $(\mathbf{n}=\mathbf{8 5})$

\begin{tabular}{|c|c|c|}
\hline & & $\begin{array}{c}P \\
\text { value }\end{array}$ \\
\hline Age, y (range) & $60.5 \pm 9(37-78)$ & $.189 *$ \\
\hline Sex, M/F & $70 / 15$ & .321 \\
\hline Obesity, no. of patients (\%) & $11(12.9)$ & $.080^{*}$ \\
\hline $\mathrm{BMI}$ & $26.0 \pm 3.1$ & .386 \\
\hline Prevalent $\mathrm{HF}$, no. of patients (\%) & $71(83.5)$ & .961 \\
\hline Angina, no. of patients $(\%)$ & $46(54.1)$ & .542 \\
\hline Isolated angina & $14(16.5)$ & .356 \\
\hline Angina + prevalent $\mathrm{HF}$ & $32(37.6)$ & .375 \\
\hline Cardiogenic shock, no. of patients $(\%)$ & $9(10.6)$ & .951 \\
\hline NYHA (Class) & $2.9 \pm 1.0$ & .612 \\
\hline NYHA class I, no. of patients $(\%)$ & $9(10.6)$ & .841 \\
\hline NYHA class II, no. of patients $(\%)$ & $20(23.5)$ & .654 \\
\hline NYHA class III, no. of patients $(\%)$ & $30(35.3)$ & .259 \\
\hline NYHA class IV, no. of patients $(\%)$ & $26(30.6)$ & .345 \\
\hline MLHF score & $44.2 \pm 21.5$ & $.153^{*}$ \\
\hline Prior cardiac surgery, no. of patients $(\%)$ & $6(7.1)$ & .987 \\
\hline PVD, no. of patients (\%) & $24(28.2)$ & .746 \\
\hline AMI, no. of patients (\%) & $82(96.5)$ & .598 \\
\hline $\mathrm{AMI}<90$ days, no. of patients $(\%)$ & $17(20.0)$ & .243 \\
\hline IABP, no. of patients $(\%)$ & $9(10.6)$ & .954 \\
\hline \multicolumn{3}{|l|}{ Echocardiographic parameters } \\
\hline LVEF, \% (range) & $27.6 \pm 6.3(14-35)$ & $.054^{*}$ \\
\hline $14 \%-19 \%$, no. of patients $(\%)$ & $8(9.5)$ & \\
\hline $20 \%-25 \%$, no. of patients $(\%)$ & $39(45.9)$ & \\
\hline $26 \%-35 \%$, no. of patients $(\%)$ & $38(44.7)$ & \\
\hline LVEDDI, $\mathrm{mm} / \mathrm{m}^{2}$ & $35.3 \pm 5.5$ & .785 \\
\hline
\end{tabular}

\begin{tabular}{lcc}
\hline & & $\begin{array}{c}\boldsymbol{P} \\
\text { value }\end{array}$ \\
\hline LVESDI, $\mathrm{mm} / \mathrm{m}^{2}$ & $28.1 \pm 5.1$ & .687 \\
LVEDVI, $\mathrm{mL} / \mathrm{m}^{2}$ & $131.6 \pm 34.7$ & .451 \\
LVESVI, $\mathrm{mL} / \mathrm{m}^{2}$ & $89.6 \pm 27.6$ & .259 \\
Mitral regurgitation (moderate-severe), & $16(18.9)$ & .958 \\
$\quad$ no. of patients (\%) & & \\
Angiography & $63.8 \pm 7.03$ & $.032^{*}$ \\
$\quad$ LVEqD & $46 / 39$ & $.005^{*}$ \\
$\quad$ Dyskinesia-akinesia & $82(96.5)$ & .942 \\
Coronary disease, no. of patients (\%) & $19(22.4)$ & .657 \\
One vessel & $16(18.8)$ & .248 \\
Two vessel & $47(55.3)$ & .364 \\
Three vessel & $13(15)$ & .855 \\
Left main stenosis, no. of patients (\%) & & \\
Intraoperative & $29(34.1)$ & .621 \\
$\quad$ Prophylactic IABP, no. of patients (\%) & $94.4 \pm 34.2$ & .254 \\
Crossclamp time & $155.3 \pm 64.6$ & .547 \\
CPB time & & \\
Medication at follow-up (65 patients) & 66 & .318 \\
$\quad \beta$-Blockers, \% & 86 & .412 \\
ACE inhibitors, \% &
\end{tabular}

$B M I$, Body mass index; $H F$, heart failure; NYHA, New York Heart Association; $M L H F$, Minnesota living with heart failure; $P V D$, peripheral vascular disease; $A M I$, acute myocardial infarction; $I A B P$, intra-aortic balloon pump; $\angle V E F$, left ventricular ejection fraction; $L V E D D I$, left ventricular enddiastolic diameter index; LVESDI, left ventricular end-systolic diameter index; LVEDVI, left ventricular end-diastolic volume index; LVESVI, left ventricular end-systolic volume index; $\angle V E q D$, left ventricular equatorial diastolic diameter; $C P B$, cardiopulmonary bypass; $A C E$, angiotensin-converting enzyme. *Variables with a $P$ value of.20 or less were selected for the final regression model. 